\title{
Germline and Tumor BRCA1/2 Pathogenic Variants in Chinese Triple-negative Breast Carcinomas
}

Gang Ji ( $\square$ jigang_2016@163.com )

Fudan University Shanghai Cancer Center https://orcid.org/0000-0001-9698-2536

\section{Longlong Bao}

Fudan University Shanghai Cancer Center

\section{Qianlan Yao}

Fudan University Shanghai Cancer Center

Jing Zhang

Fudan University Shanghai Cancer Center

\section{Xiaoli Zhu}

Fudan University Shanghai Cancer Center

\section{Qianming Bai}

Fudan University Shanghai Cancer Center

\section{Zhiming Shao}

Fudan University Shanghai Cancer Center

\section{Wentao Yang}

Fudan University Shanghai Cancer Center

Xiaoyan Zhou

Fudan University Shanghai Cancer Center

\section{Research Article}

Keywords: triple-negative breast cancer (TNBC), BRCA1/2, somatic mutation, large 97, genomic rearrangement (LRG)

Posted Date: March 11th, 2021

DOl: https://doi.org/10.21203/rs.3.rs-262082/v1

License: (a) (i) This work is licensed under a Creative Commons Attribution 4.0 International License. Read Full License

Version of Record: A version of this preprint was published at Journal of Cancer Research and Clinical Oncology on July 13th, 2021. See the published version at https://doi.org/10.1007/s00432-021-03696-2. 



\section{Dear Editors:}

We would like to submit the enclosed manuscript entitled "Germline and Tumor BRCA1/2 Pathogenic Variants in Chinese Triple-negative Breast Carcinomas", which we wish to be considered for publication in "Journal of Cancer Research and Clinical Oncology". No conflict of interest exits in the submission of this manuscript, and manuscript is approved by all authors for publication. I would like to declare on behalf of my co-authors that the work described was original research that has not been published previously, and not under consideration for publication elsewhere, in whole or in part.

The cost-effectiveness of BRCAl/2 screening for all newly diagnosed breast cancer remains controversial with respect to standard treatment. Further evidences that include incidence and outcome of BRCA1/2 pathogenic variants screened based on age or family history are needed to fully justify this conclusion. In this work, we simultaneously investigated both germline and tumor BRCA1/2 mutations including single-nucleotide variants, insertion/deletion and large genomic rearrangements, analyze their association with clinicopathological characteristics and survival outcomes in Chinese triple-negative breast cancer patients with less than or equal to 55 years. I hope this paper is suitable for "Journal of Cancer Research and Clinical Oncology".

The following is a list of possiblereviewers for your consideration: 1) Liang Cheng, Indiana University School of Medicine, liang_cheng@yahoo.com; 2) Jingjuan Yao, Department of Pathology, Memorial Sloan-Kettering Cancer Center, yaoj1 @ mskcc.org.

We deeply appreciate your consideration of our manuscript, and we look forward to receiving comments from the reviewers. If you have any queries, please don't hesitate to contact me at the address below. 
Thank you and best regards.

Yours sincerely,

First author:

Name: Gang Ji, MD

E-mail:jigang_2016@163.com

Corresponding author:

Name: Xiaoyan Zhou, phD

E-mail: xyzhou100@163.com

.

.

.


Germline and Tumor BRCA1/2 Pathogenic Variants in Chinese Triple-negative Breast Carcinomas

Gang Ji ${ }^{1,3,4 \#}$, Longlong Bao ${ }^{1,3,4 \#}$, Qianlan Yao ${ }^{1,3,4}$, Jing Zhang ${ }^{1,3,4}$, Xiaoli Zhu ${ }^{1,3,4}$, Qianming Bai ${ }^{1,3,4}$, Zhiming Shao ${ }^{2,3,4}$, Wentao Yang ${ }^{1,3,4}$, Xiaoyan Zhou ${ }^{1,3,4 *}$

${ }^{1}$ Department of Pathology, ${ }^{2}$ Department of Breast Surgery, Fudan University Shanghai

Cancer Center, Shanghai 200032, China; ${ }^{3}$ Department of Oncology, Shanghai Medical College, Fudan University, Shanghai 200032, China; ${ }^{4}$ Institute of Pathology, Fudan University, Shanghai 200032, China.

${ }^{\#}$ These authors contributed equally to this work.

*Corresponding author: Xiaoyan Zhou

Email: xyzhou100@163.com

\section{Acknowledgments}

This study was supported by Innovation Group Project of Shanghai Municipal Health Commission (Project No: 2019CXJQ03), Shanghai Science and technology development fund (Project No: 19MC1911000) and Shanghai Municipal Key Clinical Specialty (shslczdzk01301). 


\section{Abstract \\ Purpose}

The cost-effectiveness of $B R C A 1 / 2$ screening for all newly diagnosed breast cancer remains controversial with respect to standard treatment. Further evidences that include incidence and outcome of $B R C A 1 / 2$ pathogenic variants (PV) screened based on age or family history $(\mathrm{FH})$ are needed to fully justify this conclusion. We aimed to investigate germline and tumor $B R C A 1 / 2 \mathrm{PV}$ based on age screening in Chinese triple-negative breast cancer (TNBC) patients.

\section{Methods}

Paired blood and tumor DNA from 124 unselected Chinese TNBC patients with less than or equal to 55 years were collected and analyzed for BRCA1/2 PV. Clinicopathological characteristics including age at diagnosis, FH and follow-up data were collected for further analysis.

\section{Results}

The entire frequency of germline and tumor BRCA1/2 PV was $21.0 \%$ and $25 \%$, respectively. Among them, $20(16.1 \%)$ germline and $5(4.0 \%)$ somatic BRCA1/2 single-nucleotide variant/insertion/deletions were found by NGS testing, 6 (4.8\%) BRCA1 large genomic rearrangements were detected in blood DNA by MPLA. There was significant correlation between $\mathrm{FH}$ and germline BRCA1/2 $\mathrm{PV}$ among these patients. Patients with tumor $B R C A 1 / 2$ mutations had significant improvements than non-carriers in PFS $(p=0.047)$. No significant impacts were found between various mutation status in OS outcomes. No significant differences were found between 
$B R C A 1$ or BRCA2 and non-carriers in PFS or OS.

\section{Conclusion}

There is a high incidence of germline and tumor BRCA1/2 PVs in Chinese TNBC patients with less than or equal to 55 years old. Tumor $B R C A 1 / 2$ PV carriers showed an improved survival outcome. Our results suggest that BRCA1/2 PVs testing addressed within each specific clinical scenario could be more cost-effective for patients.

Keywords triple-negative breast cancer (TNBC), BRCA1/2, somatic mutation, large genomic rearrangement (LRG) . 


\section{Introduction}

Triple-negative breast cancer (TNBC) is a subtype of breast cancer lacking the expression of estrogen receptor (ER), progesterone receptor (PR) and human epidermal growth factor receptor 2 (HER2), accounting for about 15\% of all breast cancer (Denkert et al. 2017). It is characterized as aggressive, heterogeneous, and less differentiated, thereby making it difficult to intervene with a targeted therapy and often leads to poor prognosis (Bianchini et al. 2016).

The breast cancer susceptibility genes 1 and 2 (BRCA1 and BRCA2) are tumor suppressor genes involved in homologous recombination repair (HRR). Inactivation of $B R C A 1 / 2$ may lead to early onset and increase the cumulative risk of breast cancer (Miki et al. 1994; Wooster et al. 1994). A recent study estimated the average risk of developing breast cancer by age 80 years to be $72 \%$ for $B R C A 1$ and $69 \%$ for $B R C A 2$ carriers (Kuchenbaecker et al. 2017). Cells with either germline or somatic BRCAl/2 pathogenic variant (PV) in allele can survive despite the defect of HRR, but it comes at the basis of alternative repair pathway that relies heavily on poly ADP-ribose polymerase (PARP) function (Yates and Campbell 2012). Synthetic lethality effect would occur when HR deficient cells treated with PARP inhibitors (PARPi). Because of this, BRCA1/2 PV detection provides an important basis for clinical selection of PARPi therapy.

On the other hand, the cost-effectiveness of $B R C A 1 / 2$ screening for all newly diagnosed breast cancer remains controversial with respect to standard practice for preventive and therapeutic purposes (D'Andrea et al. 2016; Sun et al. 2019). Further evidence that includes the family history (FH)- or age-based prevalence of $B R C A 1 / 2$ pathogenic 
variants are needed to fully justify this conclusion. Previous studies have found that onset age was one of the clinicopathological factors associated with BRCA1/2 status, patients with $B R C A 1 / 2$ pathogenic variants were significantly younger than non-carriers (Couch et al. 2015; Lang et al. 2017; Shimelis et al. 2018). According to a survey from Chinese National Office for Cancer Prevention and Control, the peak onset age for breast cancer in Chinese women was about 50-55 years (Zuo et al. 2017). These data suggest the probability to find a high frequency of $B R C A 1 / 2 \mathrm{PV}$ in Chinese breast cancer patients under 55 years.

To our knowledge, previous studies on the prevalence of BRCA1/2 PV in TNBC mainly evaluated germline single-nucleotide variant (SNV)/insertion/deletion (indel), but usually lacked large genomic rearrangement (LGR) detection, also none specifically for patients under 55 years (Couch et al. 2015; Lang et al. 2017; Shimelis et al. 2018). Besides that, studies on tumor BRCA1/2 PV are still limited (Gonzalez-Angulo et al. 2011; Li et al. 2017; Winter et al. 2016; Zhong et al. 2016). In this study, we took advantage of next-generation sequencing (NGS) and multiplex ligation-dependent probe amplification (MLPA) technologies to simultaneously investigate germline and tumor BRCA1/2 PVs in Chinese TNBC patients with less than or equal to 55 years and analyze their association with clinicopathological factors and survival outcomes.

\section{Materials and methods}

\section{Study patients and samples}

A total of 124 female Chinese patients who got surgery in the year of 2015 in Shanghai 
Cancer Center were sequentially selected for our study population. Patients enrolled were supposed to meet the following inclusion criteria: (1) pathologically confirmed TNBC by two separate clinical pathologists (Xiaoyan Zhou, Qianming Bai). TNBC was defined as ER (-), PR (-) and HER2 (-). ER and PR status were determined by standard immunohistochemistry (IHC) method. HER2 status was determined by IHC and/or by fluorescence in situ hybridization (FISH). Results of IHC and FISH were interpreted according to NCCN guideline (Wolff et al. 2018); (2) age with 18-55 years; (3) sufficient formalin-fixed paraffin-embedded (FFPE) sections for somatic testing; and (4) willingness to provide signed consent in advance of the trial. Patients not meeting all these inclusion criteria were excluded. Blood and paired FFPE tissue samples were collected and retrieved from our biobank and the Department of Pathology for BRCA1/2 testing. Genomic DNA extracted from tumor tissue sections and peripheral blood were performed using QIAamp DNA MiniKit and QIAamp DNA MidiKit (QIAgen, Valencia, CA), respectively. Clinicopathological parameters were electronically retrieved from the Hospital Information System (HIS) of Fudan University Shanghai Cancer Center. Disease relapse or progression was determined by medical imaging, serology, or histology. Progression-free survival (PFS) was measured from diagnosis to local or systemic recurrence or the last follow-up, while OS was measured from diagnosis to death or the last follow-up. Patients have one or more family members within two generations with breast or ovarian cancer or endometrial cancer were considered having FH of HBOC. No kinship was found among them according to information of genetic counseling. The study was approved by Medical Ethics Committee of Fudan University 
Shanghai Cancer Center (Shanghai, China) and informed consent was obtained from all individual participants included in the study.

\section{BRCA1/2 SNV/indel detection by NGS}

For targeted NGS analysis, total 265 primer pairs in two pools (133 pairs in pool 1, and 132 pairs in pool 2) were used in Oncomine ${ }^{\mathrm{TM}} B R C A$ Research Assay (Thermo Fisher Scientific, Schaumburg, IL, USA), which can amplify the entire coding regions and 20 bp upstream or downstream of exon-intron boundaries of $B R C A 1 / 2$ genes. Multiplex PCR was performed using $20 \mathrm{ng}$ genomic DNA with the following cycling conditions: $99{ }^{\circ} \mathrm{C} \times 2$ minutes, 20 cycles of $99{ }^{\circ} \mathrm{C} \times 15$ seconds, and $60{ }^{\circ} \mathrm{C} \times 4$ minutes. The amplicons were treated with $2 \mu \mathrm{L}$ FuPa reagent to partially digest primers and phosphorylate the amplicons with the following conditions: $50{ }^{\circ} \mathrm{C} \times 10$ minutes, $55^{\circ} \mathrm{C} \times$ 10 minutes, and $60{ }^{\circ} \mathrm{C} \times 20$ minutes. The diluted barcodes (Thermo Fisher Scientific) were ligated with the following conditions: $22{ }^{\circ} \mathrm{C} \times 30$ minutes, and $68{ }^{\circ} \mathrm{C} \times 5$ minutes, and $72{ }^{\circ} \mathrm{C} \times 10$ minutes. Libraries were purified using Agencourt AMPure XP reagents (Beckman Coulter, Brea, CA, USA). Concentration was measured using an Ion Library Quantitation Kit (Thermo Fisher Scientific), then the same amount of $100 \mathrm{pmol} / \mathrm{L}$ libraries was pooled in one sequencing reaction. Emulsion PCR was implemented with the Ion OneTouch ${ }^{\mathrm{TM}} 2.0$ System and Hi-Q ${ }^{\mathrm{TM}}$ View OT2 reagents (Thermo Fisher Scientific) following the manufacturer's instructions. The template-positive particles were purified using Ion OneTouch ${ }^{\mathrm{TM}}$ ES system and MyOne ${ }^{\mathrm{TM}}$ Streptavidin C1 Beads (Thermo Fisher Scientific). Parallel sequencing was performed on a Personal Genome Machine (PGM) sequencer using the Ion $\mathrm{PGM}^{\mathrm{TM}} \mathrm{Hi}-\mathrm{Q}^{\mathrm{TM}}$ Sequencing Kit according to 
the manufacturer's instructions. Sequencing was performed using 500 flow runs that generated $\sim 200$ bp reads.

The sequence data were processed using standard pipeline on Torrent Suite ${ }^{\mathrm{TM}}$ version 5.4 (Thermo Fisher Scientific) as previously described (Hirotsu et al. 2015). Annotations including SNV, indel, and splice site alteration were performed using Ion Reporter ${ }^{\mathrm{TM}}$ version 5.4 (Thermo Fisher Scientific). Binary alignment map (BAM) files were visually confirmed with the Integrative Genomics Viewer (IGV) 2.4.4. Error artifacts of sequence, alignment, or variant call were discarded. Minor allele frequency (MAF) of variant less than 0.01 was considered for further pathogenicity evaluation. Variants were classified as pathogenic, likely pathogenic, uncertain significance, likely benign and benign according to ACMG guideline (Richards et al. 2015). PVs were regarded as deleterious mutations with clinical significance. MAF was identified from population database including 1000 Genomes Project database (http://phase3browser.1000genomes.org/) and dbSNP (https://www.ncbi.nlm.nih.gov/snp/) and Exome Aggregation Consortium and Exome Sequencing Project. The variants pathogenic determination referred to databases such as the BRCA Exchange database (https://brcaexchange.org/favicon.ico), LOVD database (https://databases.lovd.nl/shared/genes) and ClinVar (http:// www.ncbi.nlm.nih.gov/clinvar/). Bioinformatic tools including SIFT (http://sift.jcvi.org), Align GVGD (http://agvgd.iarc.fr/agvgd_input.php) and PolyPhen-2 (http://genetics.bwh.harvard.edu/pph2) were used as supplementary evidence to prove that a variant may affect normal function. 


\section{LGRs detection by MLPA}

Genomic DNA extracted from the patient's peripheral blood was used for BRCA1/2 LGRs detection following the MLPA instructions (MRC-Holland, Amsterdam, The Netherlands). Probe mix P002 and P087 were used for the detection or confirmation of BRCA1 LGRs. Similarly, probe mix P045 and P090 were used for BRCA2 LGRs. Briefly, 100 ng genomic DNA dissolved in $5 \mu 1$ TE buffer (Thermo Fisher Scientific) was used for denaturation with $98{ }^{\circ} \mathrm{C} \times 5$ minutes, then MLPA probe mix was added for hybridization with the following conditions: $95^{\circ} \mathrm{C} \times 1$ minute, and $60{ }^{\circ} \mathrm{C} \times 16$-20 hours. After hybridization, a Ligase-65 master mix was added for ligation with the following conditions: $54^{\circ} \mathrm{C}$ pause, $54{ }^{\circ} \mathrm{C} \times 15$ minutes, and $98{ }^{\circ} \mathrm{C} \times 5$ minutes. Multiplex PCR was performed with the following cycling conditions: 35 cycles of $95{ }^{\circ} \mathrm{C} \times 30$ seconds, $60{ }^{\circ} \mathrm{C}$ $\times 30$ seconds, $72{ }^{\circ} \mathrm{C} \times 60$ seconds and an additional $72{ }^{\circ} \mathrm{C} \times 20$ minutes. Finally, electrophoresis and data analysis were performed on ABI 3500 machine (Thermo Fisher Scientific) and Coffalyser.NET software v140721.1958 (MRC-Holland).

\section{Data analysis}

Pathologic characteristics tabulated by their types or ranges were compared between groups by chi-square test or Fisher's exact test or unpaired two-tailed t test, as appropriate. Median age at diagnosis in this cohort was 46 years (range 24-55 years). All patients were followed up until Jan-2021, or death. The median follow-up time was 50.6 months (3.1-70 months). Twelve patients were excluded because they were lost to follow-up. Survival analysis was performed using log-rank test, Kaplan-Meier analysis. 
All analyses were performed using SPSS version 19 (SPSS version 19; SPSS, Chicago, IL). $p$ values $<0.05$ were considered as statistically significant.

\section{Results}

\section{Germline and somatic BRCA1/2 PVs in TNBC}

The complete list of PVs identified in this cohort and their frequency were summarized in Table 1 and Figure 1. The entire frequency of germline and tumor BRCA1/2 PV was $21.0 \%$ and $25 \%$, respectively. All germline PVs detected by NGS were also found in paired tumor tissues. Among them, $20(16.1 \%)$ germline and $5(4.0 \%)$ somatic BRCA1/2 PVs were found by NGS testing, including 14 (11.3\%) gBRCA1, 6 (4.8\%) gBRCA2, 4 (3.2\%) sBRCA1 and $1(0.8 \%)$ sBRCA2 PVs (Figure 1A). In addition, 6 (4.8\%) BRCA1 LGRs were detected in blood DNA by MPLA, whereas no BRCA2 LGRs were found in these patients (Figure 1A). Moreover, exon 18-19 del, exon 18-20 del and c.4431 del T found in BRCA1 and c.4408_4412 del ATAAG, c.3009 del C, c.9019 A>T, c.4657_4658 del AC found in BRCA2 had not been previously reported and therefore, are novel (Table 1).

All BRCA1/2 PVs including SNV, indel and LGRs were visualized by IGV software or Coffalyser software. Notablely, no patient in this cohort was found with two or more deleterious PVs showing a mutual exclusion of $B R C A 1 / 2 \mathrm{PV}$. In terms of distribution, all PVs are scattered in various functional domains and protein binding regions of $B R C A 1 / 2$ (Figure 2). 


\section{The association between $B R C A 1 / 2$ PVs and clinicopathological characteristics}

Age, FH of HBOC, histology, tumor grade, TNM stage and ki67 expression were included in pathologic characteristics. The median age in this cohort was 46 years (range 24-55 years). A minority of patients (12.9\%) had potentially significant $\mathrm{FH}$, and $92.7 \%$ of them were invasive ductal cancer (IDC) with tumor grade of 2 or 3 . The proportion of both $B R C A 1 / 2$ germline $(50 \%)$ and tumor $\mathrm{PV}(50 \%)$ in the patients with $\mathrm{FH}$ are significantly higher than that in those without $\mathrm{FH}(16.7 \%$ and $21.3 \%)$ (all $P<0.05)$. No significant association was found between $B R C A 1 / 2$ status and clinicopathological characteristics including age, histology, grade, stage and ki67 expression by chi-square test (Table 2). However, Patients with BRCAl LGRs tended to be younger when compared with non-carriers (mean age 39 vs. 45.5 years, $p=0.025$ ) and $B R C A 1$ SNV/indel PV ones (mean age 39 vs. 45.9 years, $p=0.035$, Supplementary material) by unpaired two-tailed t test.

\section{Survival analysis}

At a median follow-up time of 50.6 months (3.1-70 months), there were 17/112 (15.2\%) recurrences and 10/112 (8.9\%) deaths in this cohort. The Kaplan Meier plots for PFS and OS by mutation are shown in Figure 3-4. Patients with tumor BRCA1/2 mutations had significant improvements than non-carriers in PFS (Figure 3, $p=0.047$ ). No significant impacts were found between various mutation status in OS outcomes. No significant differences were found between BRCA1 or BRCA2 and non-carriers in PFS or OS (Figure 4). The low number of events precluded Cox regression analysis. 


\section{Discussion}

In this study, 124 paired tumor and peripheral blood samples from Chinese TNBC patients with less than or equal to 55 years were analyzed for the entire coding regions and exon-intron boundaries of $B R C A 1 / 2$. This study provided the first simultaneous overview of the germline and tumor BRCA1/2 PVs including SNV, indel and LGRs in Chinese TNBC patients.

Studies on the spectrum and prevalence of BRCA1/2 PVs among TNBC patients have been reported over the years. There have been two large-scale germline BRCA1/2 screening in unselected TNBC cohort in the United States, the prevalence of PVs (SNV and indel) was 8.4\% (714/8753) and 10.4\% (224/2148), respectively (Couch et al. 2015; Shimelis et al. 2018). In China, a multicenter research on germline BRCA1/2 PV (SNV and indel) was performed in 2991 unselected breast carcinomas, they found an overall incidence of $10.1 \%$ among 1009 TNBC subgroup (Lang et al. 2017). For somatic PV, the limited studies to date showed that $B R C A 1 / 2 \mathrm{PV}$ rate in TNBC ranged from $1.3 \%$ to $1.43 \%$ (Gonzalez-Angulo et al. 2011; Zhong et al. 2016). In our current work, the incidence of germline and somatic BRCA1/2 PV (SNV and indel) was $16.1 \%$ and $4 \%$, respectively, which was a little bit higher than the above studies. One of the reasons for the difference is the deviation that may be caused by our small sample size. Another equally important reason is that the onset age of patients selected in this cohort was less than or equal to 55 years. Since Couch et al. also found that the germline BRCA1/2 PV rate were $16.6 \%$ $(125 / 754)$ and $14.3 \%(160 / 1120)$ in patients under 50 years and 60 years, respectively, 
which were significantly higher than that in patients without regard to age $10.4 \%$ (224/2148) (Couch et al. 2015). Our finding suggests a high BRCA1/2 PV frequency in Chinese TNBC patients under 55 years. Moreover, we also found a significantly higher proportion of both germline and tumor $B R C A 1 / 2 \mathrm{PVs}$ in the patients with $\mathrm{FH}$ than that in sporadic patients, which provides a basis for the hypothesis that BRCA1/2 PVs testing addressed within each specific clinical scenario could be more cost-effective for patients. Although the PARPi olaparib is now approved in advanced germline BRCA1/2 breast cancer, this treatment may remain unaffordable to many health care systems and patients for many years (Tutt et al. 2018). For most patients, chemotherapy remains the only available non-investigational systemic treatment option for non-BRCA-mutated advanced TNBC (Cardoso et al. 2020). There is no specific recommendation on drug types, except for platinum compounds for patients with BRCA1/2 mutations (Cardoso et al. 2020). A randomized phase III trial comparing carboplatin with the microtubule-disrupting agent docetaxel in advanced unselected TNBC, they found that patients with germline BRCA1/2 PVs showed a significant advantage in objective response rate and PFS with platinum therapy compared to docetaxel (Tutt et al. 2018). In this study, we also found that $B R C A 1 / 2 \mathrm{PV}$ carriers responded better to systemic treatment. Our results support the importance of BRCA1/2 testing in clinical decision-making of TNBC.

There are still some deficiencies in this work. Firstly, as we mentioned earlier, the sample size we screened is relatively small, which may lead to partial deviation of PV frequency. However, this deficiency has been verified by comparing with the results of large-scale domestic and international studies, which proves that the BRCA1/2 PV 
frequencies we obtained by age-based screening are highly reliable. Secondly, although 4.8\% LGRs have been detected in blood samples by MLPA, we did not detect LGRs in tumor samples. Because FFPE or fresh tissue sample are difficult technical challenge for MLPA method, here we make assumptions that all LGRs identified from the blood samples are also present in the tumor tissues based on theory. Finally, HR genes includes Fanconi anemia genes (BRIP1, PALB2), the core $R A D$ genes $(R A D 51 C, R A D 51 D)$, and genes involved in HR pathways either directly $(C H E K 2, B A R D 1, N B N, A T M)$ or indirectly (CDK12), but is not limited to BRCA1/2 (Boussios et al. 2020). Multigene panel testing may find more potential beneficiaries, it should be attempted in future work.

In summary, there is a high incidence of germline (21.0\%) and tumor (25.0\%) BRCA1/2 PVs in Chinese TNBC patients with less than or equal to 55 years old. LGRs and somatic PV detection should be considered in chemotherapy or targeted therapy in TNBC. Tumor BRCAl/2 PV carriers showed an improved survival outcome, which indicate that comprehensive detection of $B R C A 1 / 2 \mathrm{PV}$, including SNV, indel and LGRs, would benefit more patients. Our results suggest that BRCA1/2 PVs testing addressed within each specific clinical scenario could be more cost-effective for patients.

\section{Acknowledgments}

This study was supported by Innovation Group Project of Shanghai Municipal Health Commission (Project No: 2019CXJQ03), Shanghai Science and technology development 
fund (Project No: 19MC1911000) and Shanghai Municipal Key Clinical Specialty (shslczdzk01301).

\section{Declarations}

Funding This study was supported by Innovation Group Project of Shanghai Municipal Health Commission (Project No: 2019CXJQ03), Shanghai Science and technology development fund (Project No: 19MC1911000) and Shanghai Municipal Key Clinical Specialty (shslczdzk01301).

Conflicts of interest The authors have no conflicts of interest to declare.

Availability of data and material All raw data can be viewed in NODE (http://www.biosino.org/node) by pasting the accession OEP000931 into the text search box or through the URL: http://www.biosino.org/node/project/detail/OEP000931.

Code availability Not applicable.

\section{Author contributions}

(I) Conception and design: Xiaoyan Zhou

(II) Administrative support: Xiaoyan Zhou

(III) Provision of study materials or patients: Xiaoli Zhu, Qianming Bai, Zhiming Shao and Wentao Yang

(IV) Collection and assembly of data: Gang Ji, Longlong Bao and Jing Zhang

(V) Data analysis and interpretation: Gang Ji and Qianlan Yao

(VI) Manuscript writing: All authors

(VII) Final approval of manuscript: All authors 
Ethics approval The study was approved by Medical Ethics Committee of Fudan University Shanghai Cancer Center (Shanghai, China).

Consent to participate Informed consent was obtained from all individual participants included in the study.

Consent for publication Not applicable.

\section{References}

Bianchini G, Balko JM, Mayer IA, Sanders ME, Gianni L (2016) Triple-negative breast cancer: challenges and opportunities of a heterogeneous disease Nat Rev Clin Oncol 13:674-690 doi:10.1038/nrclinonc.2016.66

Boussios S et al. (2020) Wise Management of Ovarian Cancer: On the Cutting Edge Journal of personalized medicine 10 doi:10.3390/jpm10020041 Cardoso F et al. (2020) 5th ESO-ESMO international consensus guidelines for advanced breast cancer (ABC 5) Ann Oncol 31:1623-1649 doi:10.1016/j.annonc.2020.09.010

Couch FJ et al. (2015) Inherited mutations in 17 breast cancer susceptibility genes among a large triple-negative breast cancer cohort unselected for family history of breast cancer J Clin Oncol 33:304-311 doi:10.1200/JCO.2014.57.1414

D'Andrea E, Marzuillo C, De Vito C, Di Marco M, Pitini E, Vacchio MR, Villari P (2016) Which BRCA genetic testing programs are ready for implementation in health care? A systematic review of economic evaluations Genetics in medicine : official journal of the American College of Medical Genetics 18:1171-1180 doi:10.1038/gim.2016.29

Denkert C, Liedtke C, Tutt A, von Minckwitz G (2017) Molecular alterations in triple-negative breast cancer-the road to new treatment strategies Lancet 389:2430-2442 doi:10.1016/S0140-6736(16)32454-0

Gonzalez-Angulo AM et al. (2011) Incidence and outcome of BRCA mutations in unselected patients with triple receptor-negative breast cancer Clin Cancer Res 17:1082-1089 doi:10.1158/1078-0432.CCR-10-2560

Hirotsu Y, Nakagomi H, Sakamoto I, Amemiya K, Oyama T, Mochizuki H, Omata M (2015) Multigene panel analysis identified germline mutations of DNA repair genes in breast and ovarian cancer Mol Genet Genomic Med 3:459-466 doi:10.1002/mgg3.157

Kuchenbaecker KB et al. (2017) Risks of Breast, Ovarian, and Contralateral Breast Cancer for BRCA1 and BRCA2 Mutation Carriers Jama 317:2402-2416 doi:10.1001/jama.2017.7112

Lang GT et al. (2017) The spectrum of BRCA mutations and characteristics of BRCA-associated breast cancers in China: Screening of 2,991 patients and 1,043 controls by next-generation sequencing Int J Cancer 141:129-142 doi:10.1002/ijc.30692

Li G et al. (2017) Analysis of BRCA1/2 mutation spectrum and prevalence in unselected Chinese breast cancer patients by next-generation sequencing J Cancer Res Clin Oncol 143:2011-2024 doi:10.1007/s00432-017-2465-8

Miki Y et al. (1994) A strong candidate for the breast and ovarian cancer susceptibility gene BRCA1 Science 266:66-71 doi:10.1126/science.7545954 Richards S et al. (2015) Standards and guidelines for the interpretation of sequence variants: a joint consensus recommendation of the American College of Medical Genetics and Genomics and the Association for Molecular Pathology Genet Med 17:405-424 doi:10.1038/gim.2015.30 Shimelis $\mathrm{H}$ et al. (2018) Triple-Negative Breast Cancer Risk Genes Identified by Multigene Hereditary Cancer Panel Testing J Natl Cancer Inst 110:855-862 doi:10.1093/jnci/djy106

Sun L et al. (2019) A Cost-effectiveness Analysis of Multigene Testing for All Patients With Breast Cancer JAMA oncology doi:10.1001/jamaoncol.2019.3323 Tutt A et al. (2018) Carboplatin in BRCA1/2-mutated and triple-negative breast cancer BRCAness subgroups: the TNT Trial Nat Med 24:628-637 doi:10.1038/s41591-018-0009-7 
Winter C et al. (2016) Targeted sequencing of BRCA1 and BRCA2 across a large unselected breast cancer cohort suggests that one-third of mutations are somatic Ann Oncol 27:1532-1538 doi:10.1093/annonc/mdw209

Wolff AC et al. (2018) Human Epidermal Growth Factor Receptor 2 Testing in Breast Cancer: American Society of Clinical Oncology/College of American Pathologists Clinical Practice Guideline Focused Update J Clin Oncol 36:2105-2122 doi:10.1200/JCO.2018.77.8738

Wooster R et al. (1994) Localization of a breast cancer susceptibility gene, BRCA2, to chromosome 13q12-13 Science 265:2088-2090 doi:10.1126/science.8091231 Yates LR, Campbell PJ (2012) Evolution of the cancer genome Nat Rev Genet 13:795-806 doi:10.1038/nrg3317

Zhong X et al. (2016) Prevalence and Prognostic Role of BRCA1/2 Variants in Unselected Chinese Breast Cancer Patients PLoS One 11:e0156789 doi:10.1371/journal.pone.0156789

Zuo TT, Zheng RS, Zeng HM, Zhang SW, Chen WQ (2017) Female breast cancer incidence and mortality in China, 2013 Thorac Cancer 8:214-218 doi:10.1111/1759-7714.12426

0

1

2

323

4




\section{Figures and Figure legends}
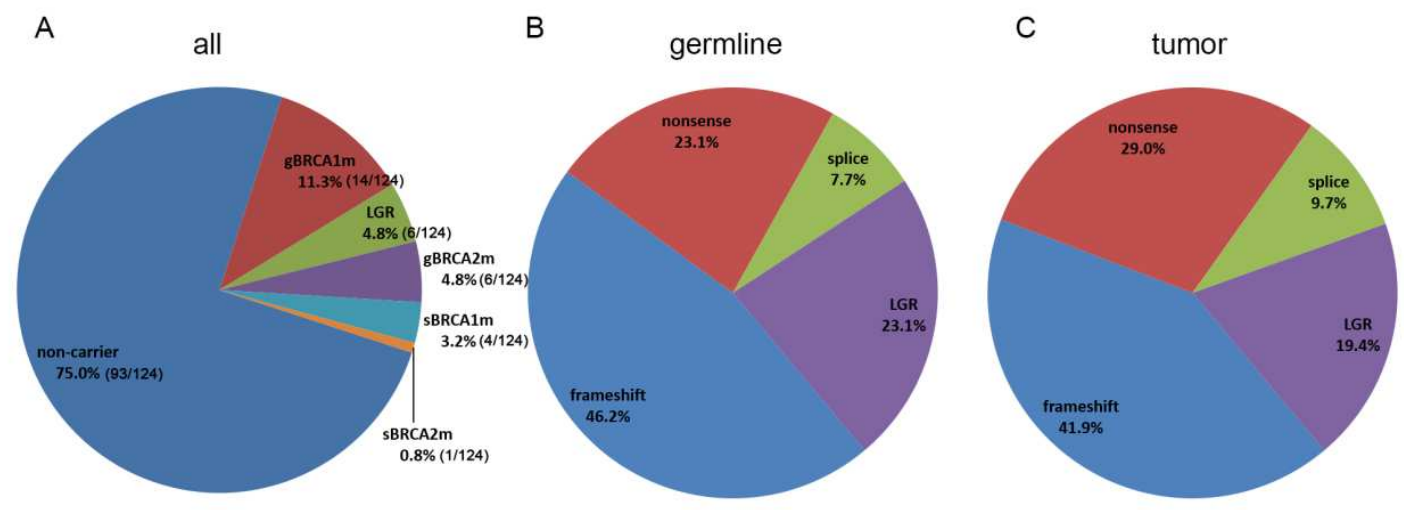

Figure 1. Summary of BRCA1/2 PVs in 124 TNBC patients. (A) Distribution of all PVs and frequencies. (B) Proportion of germline PV types detected in blood DNA. (C) Proportion of tumor PV types detected in tumor DNA. $g B R C A 1 / 2 m$ germline $B R C A 1 / 2$ $\mathrm{SNV} /$ Indel, $L R G$ Large genomic rearrangement, $s B R C A 1 / 2 m$ somatic $B R C A 1 / 2$ SNV/Indel. 

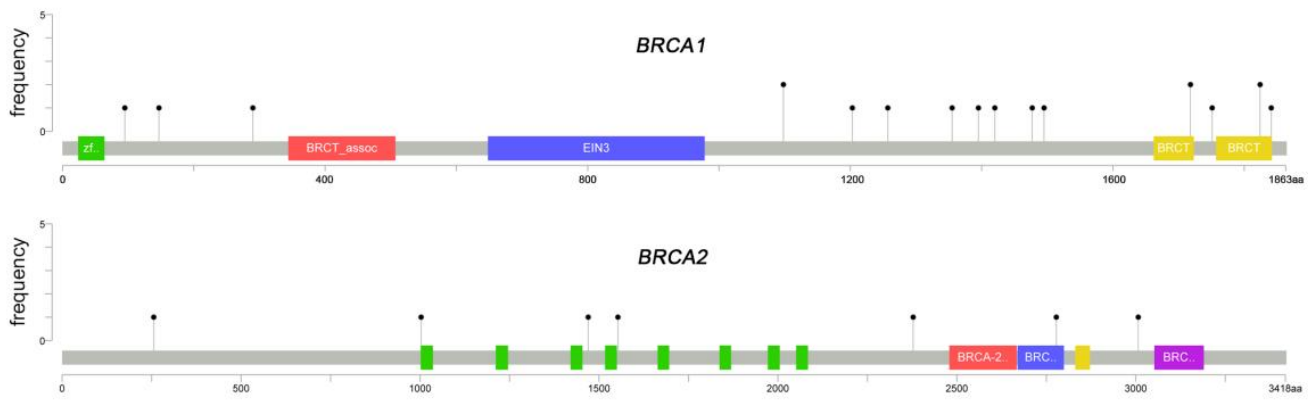

455

Figure 2. Schematic representation of $B R C A 1 / 2 \mathrm{PVs}$ in functional domains and protein binding regions. 
A

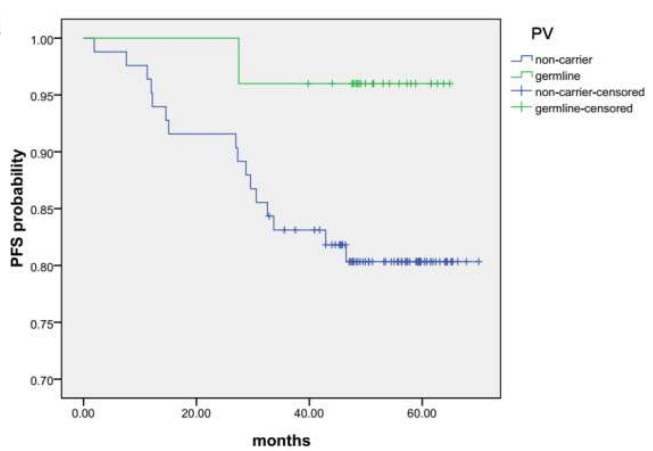

C

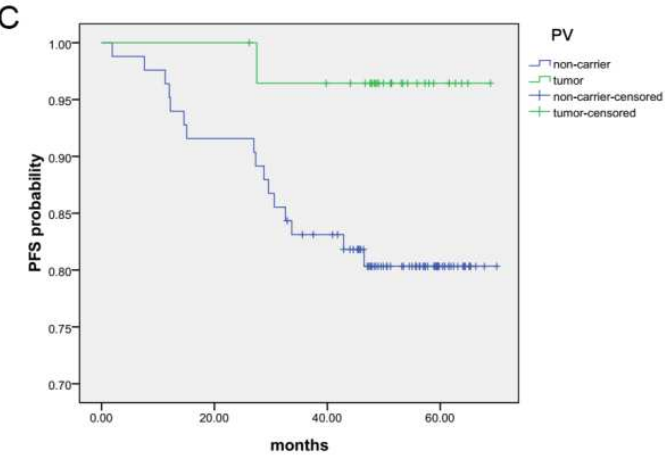

B

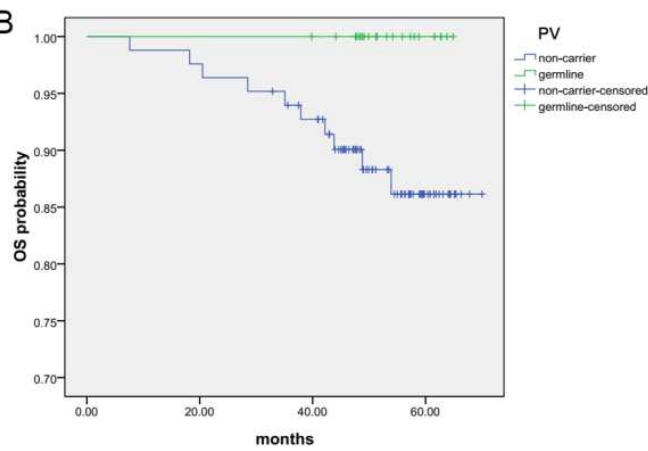

D

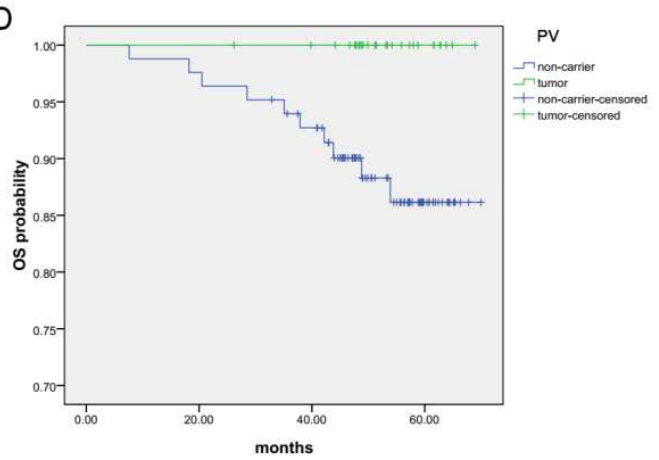

474

Figure 3. Kaplan-Meier survival analysis on germline and tumor $B R C A 1 / 2$ PVs in TNBC. (A) Progression-free survival (PFS) with and without germline PV. (B) Overall survival (OS) with and without germline PV. (C) PFS with and without tumor PV. (D) OS with and without tumor PV. 
A

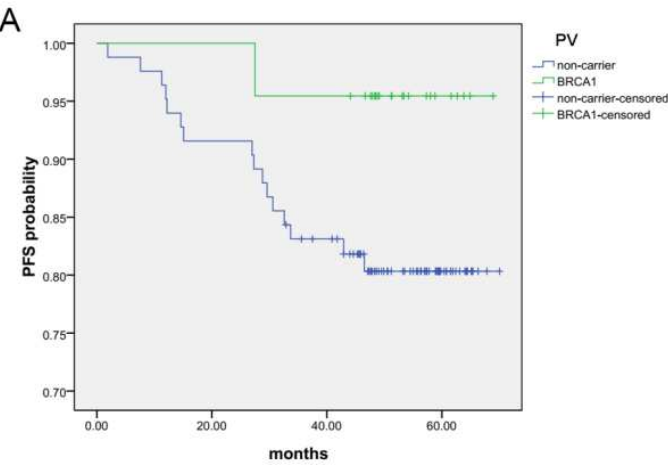

C

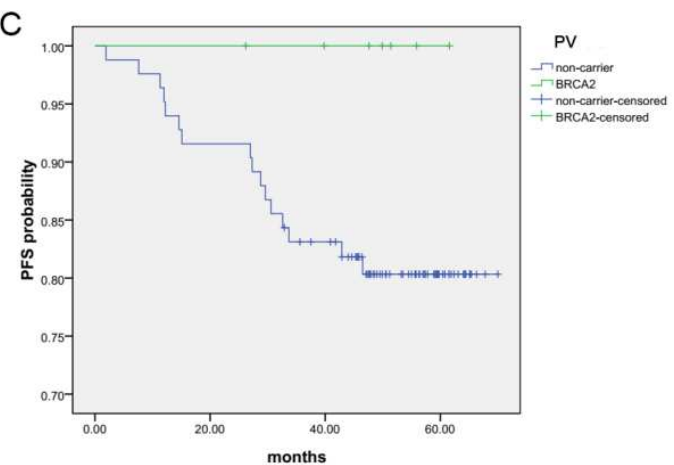

B

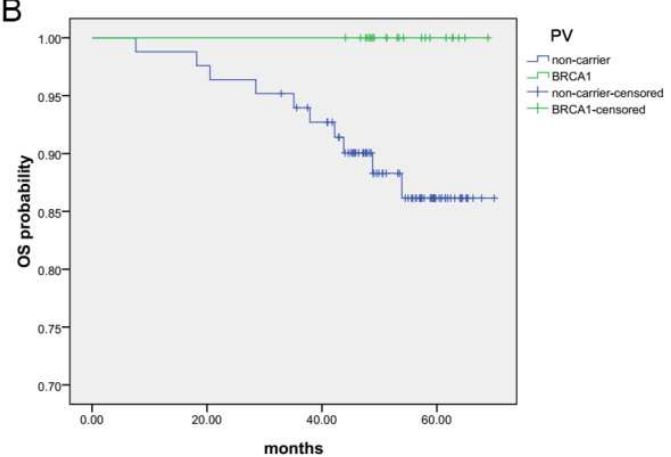

D

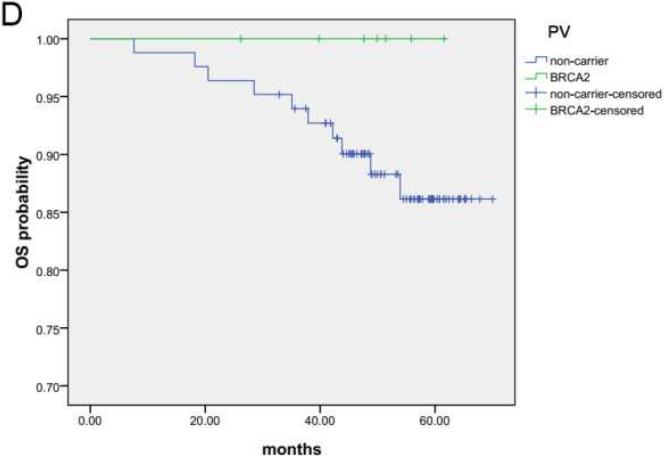

488

Figure 4. Kaplan-Meier survival analysis on various BRCA1/2 PVs in TNBC. (A) Progression-free survival (PFS) with and without BRCAl PV. (B) Overall survival (OS) with and without BRCA1 PV. (C) PFS with and without BRCA2 PV. (D) OS with and without $B R C A 2 \mathrm{PV}$. 

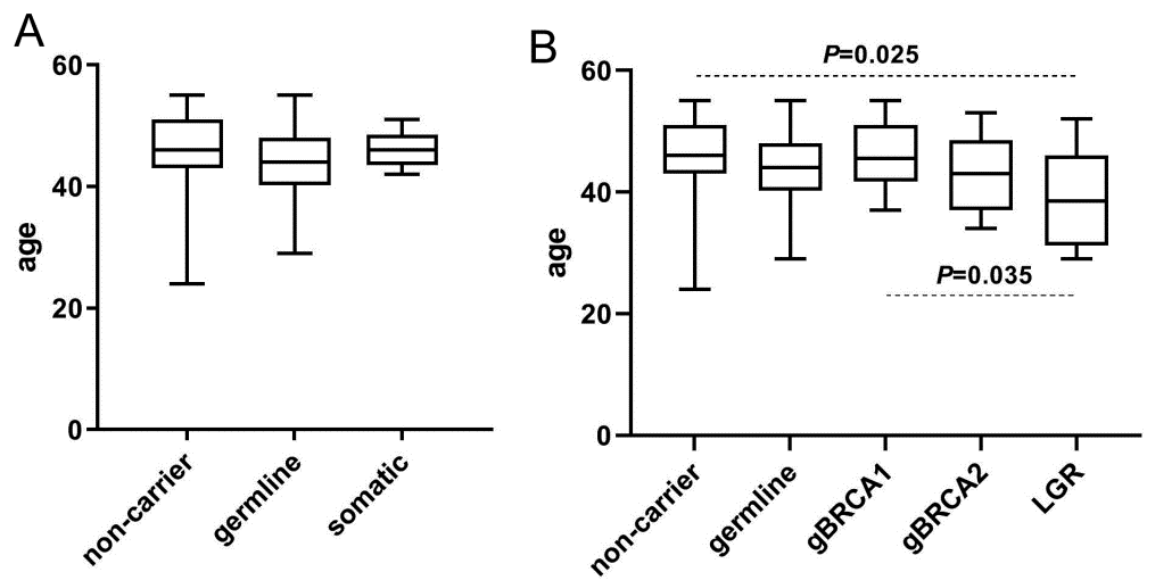

Supplement material Comparison of ages among groups. Groups were classified by (A) sample types and (B) PV types. $p$ values calculated using unpaired two-tailed t test. 
515 Table 1 The list of patients with $B R C A 1 / 2$ pathogenic variant and related information

\begin{tabular}{|c|c|c|c|c|c|c|c|c|c|c|}
\hline No & age/sex & $\mathrm{FH}$ & grade & stage & Gene & $\begin{array}{l}\text { Exon/intro } \\
n\end{array}$ & Variation & AA Change & Variant effect & type \\
\hline 1 & $46 / F$ & no & 3 & $\| \mathrm{b}$ & $B R C A 1$ & Exon 6 & c.283_286delCTTG & p.(Leu95Thrf^*23) & Frameshift & germline \\
\hline 2 & $52 / F$ & no & 3 & I a & $B R C A 1$ & Exon 11b & c.3294delT & p.(Pro1099Leufs*10) & Frameshift & germline \\
\hline 3 & $55 / F$ & no & 2 & $\| \mathrm{b}$ & $B R C A 1$ & Exon 11b & c.3294delT & p.(Pro1099Leufs*10) & Frameshift & germline \\
\hline 4 & $45 / F$ & yes & 2 & I a & $B R C A 1$ & Exon 11b & c. $3607 \mathrm{C}>\mathrm{T}$ & p. $(\operatorname{Arg} 1203 *)$ & Nonsense & germline \\
\hline 5 & $51 / F$ & no & 3 & I a & $B R C A 1$ & Exon 11b & c.3770_3771delAG & p.(Glu1257Glyfs*9) & Frameshift & germline \\
\hline 6 & $41 / F$ & no & 3 & I a & $B R C A 1$ & Exon $11 b$ & c.4065_4068delTCAA & p.(Asn1355Lysfs*10) & Frameshift & germline \\
\hline 7 & $41 / F$ & yes & 3 & $\| \mathrm{b}$ & $B R C A 1$ & Intron 7 & c. $441+2 T>C$ & - & splice & germline \\
\hline 8 & $46 / F$ & no & 3 & I a & $B R C A 1$ & Intron 14a & c. $4485-2 A>C$ & - & splice & germline \\
\hline 9 & $42 / F$ & no & 3 & I a & $B R C A 1$ & Exon 19 & c. $5154 \mathrm{G}>\mathrm{A}$ & p.(Trp1718*) & Nonsense & germline \\
\hline 10 & $45 / F$ & yes & 3 & $\| \mathrm{a}$ & $B R C A 1$ & Exon 19 & c. $5154 \mathrm{G}>\mathrm{A}$ & p.(Trp1718*) & Nonsense & germline \\
\hline 11 & $37 / F$ & no & 3 & $\| \mathrm{a}$ & $B R C A 1$ & Exon 24 & c.5470_5477delATTGGGCA & p.(lle1824Aspfs $\star 3)$ & Frameshift & germline \\
\hline 12 & $51 / F$ & no & 3 & I a & $B R C A 1$ & Exon 24 & c.5470_5477delATTGGGCA & p.(lle1824Aspfs $\star 3)$ & Frameshift & germline \\
\hline 13 & $43 / F$ & no & 2 & I a & $B R C A 1$ & Exon 24 & c.5521delA & p.(Ser1841Valfs*2) & Frameshift & germline \\
\hline 14 & $47 / F$ & yes & 2 & I a & $B R C A 1$ & Exon $11 b$ & c.869delT & p.(Leu290Tyrfs*8) & Frameshift & germline \\
\hline 15 & $38 / F$ & no & 3 & II a & $B R C A 2$ & Exon 14 & c. $7133 C>G$ & p.(Ser2378*) & Nonsense & germline \\
\hline 16 & $47 / F$ & no & 2 & $\mathrm{II}$ a & $B R C A 2$ & Exon 9 & c.771_775delTCAAA & p.(Asn257Lysfs*17) & Frameshift & germline \\
\hline 17 & $44 / F$ & no & 3 & $\mathrm{II}$ a & $B R C A 2$ & Exon 11 & c.3009delC & p. $($ His1003GInfs*40) & Frameshift & Germline\# \\
\hline 18 & $53 / F$ & yes & 3 & $\mathrm{II}$ a & BRCA2 & Exon 11 & c.4408_4412delATAAG & p.(Ile1470Lysfs*10) & Frameshift & germline\# \\
\hline 19 & $34 / F$ & no & 3 & $\| \mathrm{b}$ & $B R C A 2$ & Exon 11 & c.4657_4658delAC & p.(Thr1553*) & Nonsense & germline\# \\
\hline 20 & $42 / F$ & yes & 3 & $\| \mathrm{b}$ & $B R C A 2$ & Exon 23 & c. $.9019 A>T$ & p. $(\operatorname{Arg} 3007 *)$ & Nonsense & germline \\
\hline 21 & $29 / F$ & yes & 3 & II a & $B R C A 1$ & - & exon $18-20$ del & - & LGR & germline\# \\
\hline 22 & $36 / F$ & yes & 3 & $\| \mathrm{a}$ & $B R C A 1$ & - & exon $18-20$ del & - & LGR & germline\# \\
\hline 23 & $52 / F$ & no & 3 & I a & $B R C A 1$ & - & exon 8-9 del & - & LGR & germline \\
\hline 24 & $32 / F$ & no & 3 & IIIa & $B R C A 1$ & - & exon 3 del & - & LGR & germline\# \\
\hline 25 & $41 / F$ & no & 3 & $\mathrm{II}$ a & $B R C A 1$ & - & exon 18-19 del & - & LGR & germline\# \\
\hline 26 & $44 / F$ & no & - & $\| \mathrm{b}$ & $B R C A 1$ & - & exon 11 del & - & LGR & germline \\
\hline 27 & $51 / F$ & no & 2 & II a & $B R C A 1$ & Exon 12 & c. $4183 C>T$ & p. $(G \ln 1395 *)$ & Nonsense & somatic \\
\hline 28 & $42 / F$ & no & 3 & IIIa & $B R C A 1$ & Exon 20 & c. $5251 \mathrm{C}>\mathrm{T}$ & p. $(\operatorname{Arg} 1751 *)$ & Nonsense & somatic \\
\hline 29 & $45 / F$ & no & 2 & I a & $B R C A 1$ & Exon 13 & c. $4258 \mathrm{C}>\mathrm{T}$ & p. $(G \ln 1420 *)$ & Nonsense & somatic \\
\hline 30 & $46 / F$ & no & 3 & $\| \mathrm{b}$ & $B R C A 2$ & Intron 18 & c. $8332-2 A>C$ & - & splice & somatic \\
\hline 31 & $46 / F$ & no & 3 & IIIa & $B R C A 1$ & Exon $14 a$ & c.4431delT & p.(Phe1477Leufs $\star 28)$ & Frameshift & somatic\# \\
\hline
\end{tabular}

\begin{tabular}{ll}
\hline 516 & $F H$ family history \\
517 & $A A$ amino acid \\
518 & $F$ female \\
519 & $L G R$ large genomic rearrangement \\
520 & $\#$ novel
\end{tabular}

521 
Table 2. The association between $B R C A 1 / 2$ pathogenic variants and clinicopathologic characteristics

\begin{tabular}{|c|c|c|c|c|c|c|c|c|}
\hline \multirow{2}{*}{ Characteristics } & \multirow{2}{*}{ non-carrier } & \multicolumn{6}{|c|}{ pathogenic variants } & \multirow[b]{2}{*}{$p^{\mathrm{d}}$} \\
\hline & & tumor & $p^{\mathrm{a}}$ & germline & $p^{\mathrm{b}}$ & somatic & $p^{\mathrm{c}}$ & \\
\hline \multicolumn{2}{|c|}{ family history of $\mathrm{HBOC}$} & & 0.026 & & 0.007 & & 1 & 0.245 \\
\hline Yes (16) & $8(50 \%)$ & $8(50 \%)$ & & $8(50 \%)$ & & 0 & & \\
\hline No (108) & $85(78.7 \%)$ & $23(21.3 \%)$ & & $18(16.7 \%)$ & & $5(4.6 \%)$ & & \\
\hline age & & & 1.000 & & 0.676 & & 0.581 & 0.553 \\
\hline$\leq 40 \quad(24)$ & $18(75 \%)$ & $6(25 \%)$ & & $6(25 \%)$ & & 0 & & \\
\hline $41-55(100)$ & $75(75 \%)$ & $25(25 \%)$ & & $20(20 \%)$ & & $5(5 \%)$ & & \\
\hline histology & & & 0.448 & & 0.682 & & 1 & 1 \\
\hline IDC (115) & $85(73.9 \%)$ & $30(26.1 \%)$ & & $25(21.7 \%)$ & & $5(4.3 \%)$ & & \\
\hline others $(9)^{\mathrm{e}}$ & $8(88.9 \%)$ & $1(11.1 \%)$ & & $1(11.1 \%)$ & & 0 & & \\
\hline grade & & & 0.646 & & 0.807 & & 0.552 & 1 \\
\hline $2(25)$ & $18(72 \%)$ & $7(28 \%)$ & & $5(20 \%)$ & & $2(8 \%)$ & & \\
\hline $3(90)$ & $67(74.4 \%)$ & $23(25.6 \%)$ & & $20(22.2 \%)$ & & $3(3.3 \%)$ & & \\
\hline unclassified (9) & $8(88.9 \%)$ & $1(11.1 \%)$ & & $1(11.1 \%)$ & & 0 & & \\
\hline stage & & & 0.473 & & 0.241 & & 0.17 & 1 \\
\hline 0/I（53） & $42(79.2 \%)$ & $11(20.8 \%)$ & & $10(18.9 \%)$ & & $1(1.9 \%)$ & & \\
\hline II （56） & $39(69.6 \%)$ & $17(30.4 \%)$ & & $15(26.8 \%)$ & & $2(3.6 \%)$ & & \\
\hline III（15） & $12(80 \%)$ & $3(20 \%)$ & & $1(6.7 \%)$ & & $2(13.3 \%)$ & & \\
\hline ki67 & & & 0.124 & & 0.187 & & 0.588 & 0.271 \\
\hline$\leq 30 \% （ 26 ）$ & $23(88.5 \%)$ & $3(11.5 \%)$ & & $3(11.5 \%)$ & & 0 & & \\
\hline$>30 \%(98)$ & $70(71.4 \%)$ & $28(28.6 \%)$ & & $23(23.5 \%)$ & & $5(5.1 \%)$ & & \\
\hline
\end{tabular}

$a$ tumor vs. non-carrier

$b$ gemline vs. non-carrier

$c$ somatic vs. non-carrier

$d$ germline vs. somatic

$e$ others contain 4 metaplastic carcinoma, 2 lobular carcinoma, 1 adenoid cystic carcinoma, 1 apocrineductal carcinoma in situ and 1 encapsulated papillary carcinoma $p$ values are calculated by chi-square test or Fisher's exact test, as appropriate $I D C$ invasive ductal carcinoma 


\section{Figures}
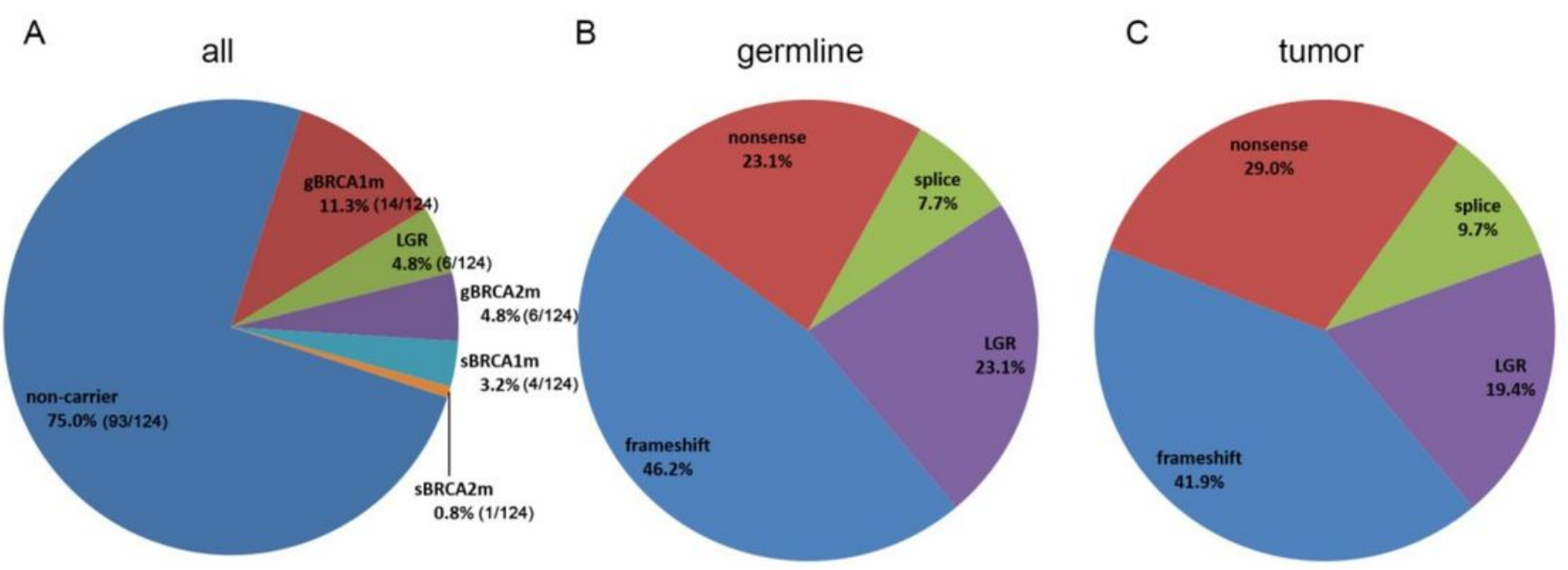

\section{Figure 1}

Summary of BRCA1/2 PVs in 124 TNBC patients. (A) Distribution of all PVs and frequencies. (B) Proportion of germline PV types detected in blood DNA. (C) Proportion of tumor PV types detected in tumor DNA. gBRCA1/2m germline BRCA1/2 SNV/Indel, LRG Large genomic rearrangement, sBRCA1/2m somatic BRCA1/2 SNV/Indel.
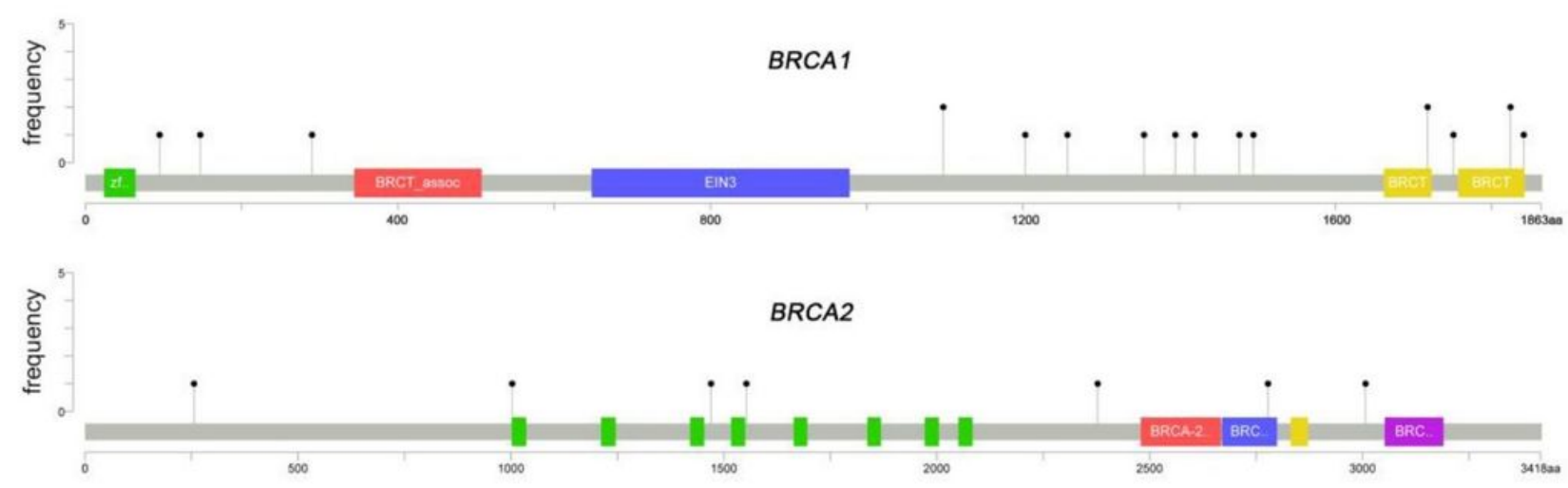

Figure 2

Schematic representation of BRCA1/2 PVs in functional domains and protein binding regions. 

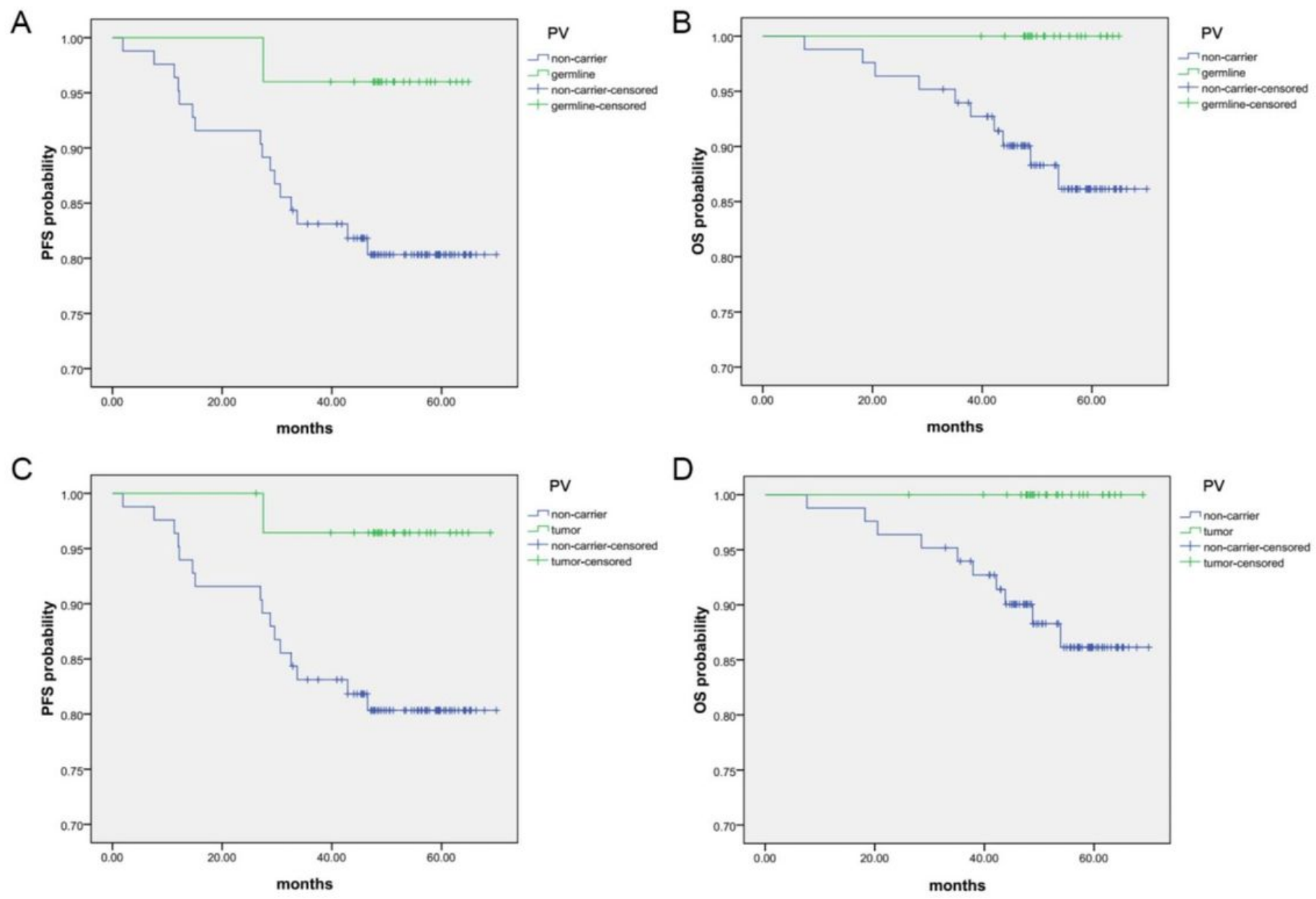

\section{Figure 3}

Kaplan-Meier survival analysis on germline and tumor BRCA1/2 PVs in TNBC. (A) Progression-free survival (PFS) with and without germline PV . (B) Overall survival (OS) with and without germline PV . (C) PFS with and without tumor PV. (D) OS with and without tumor PV. 
A

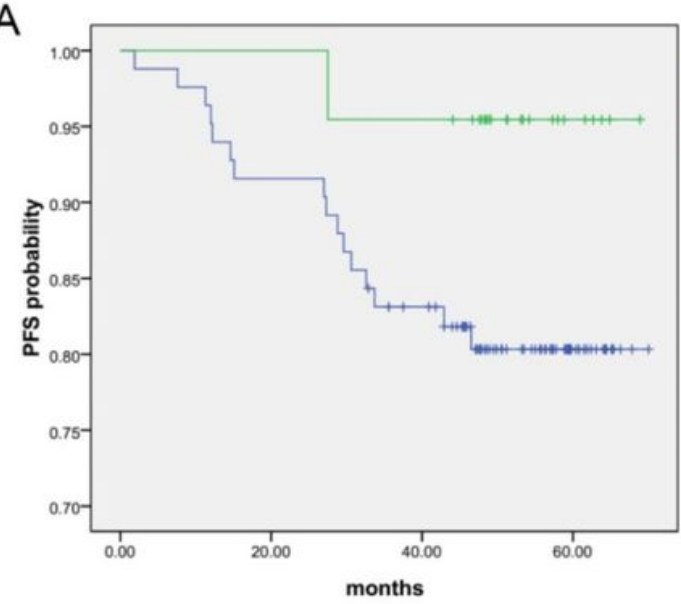

C

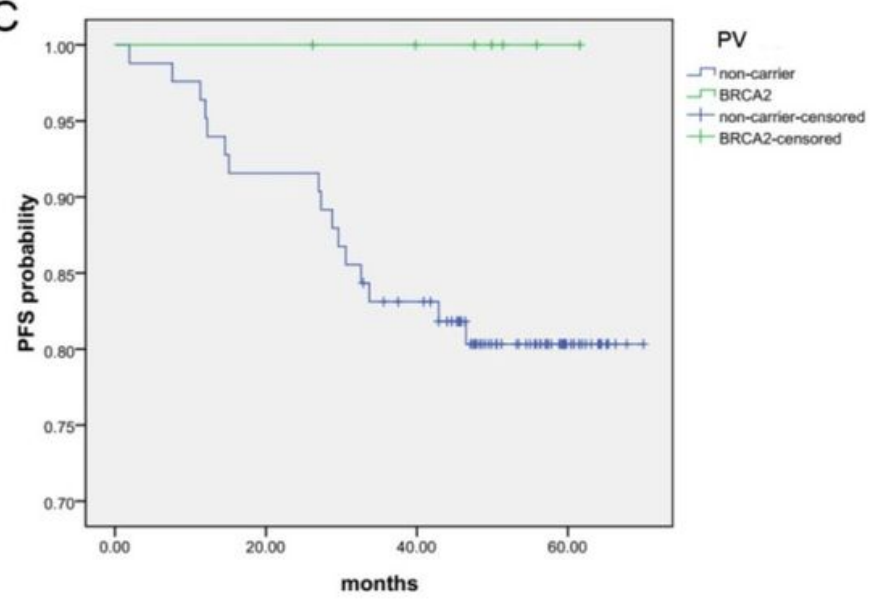

B

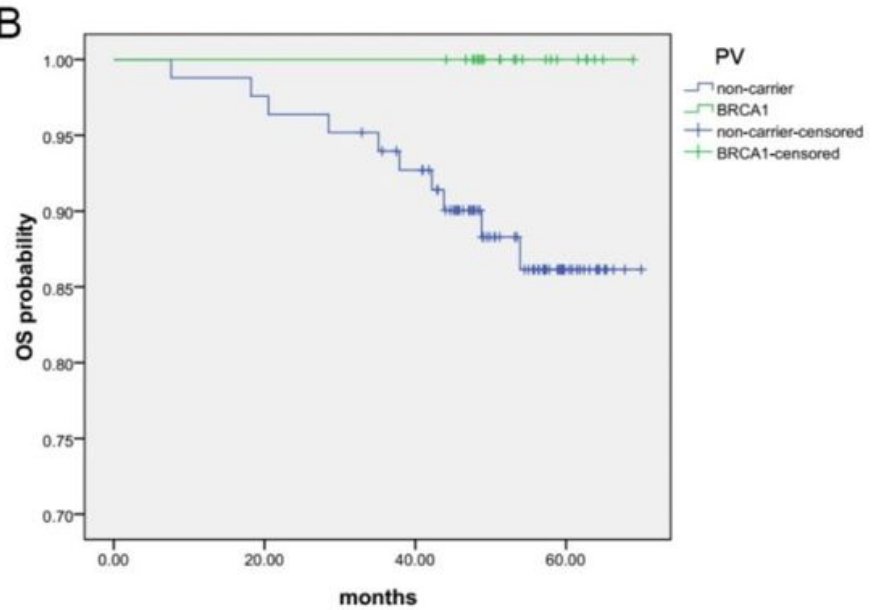

$\mathrm{D}$

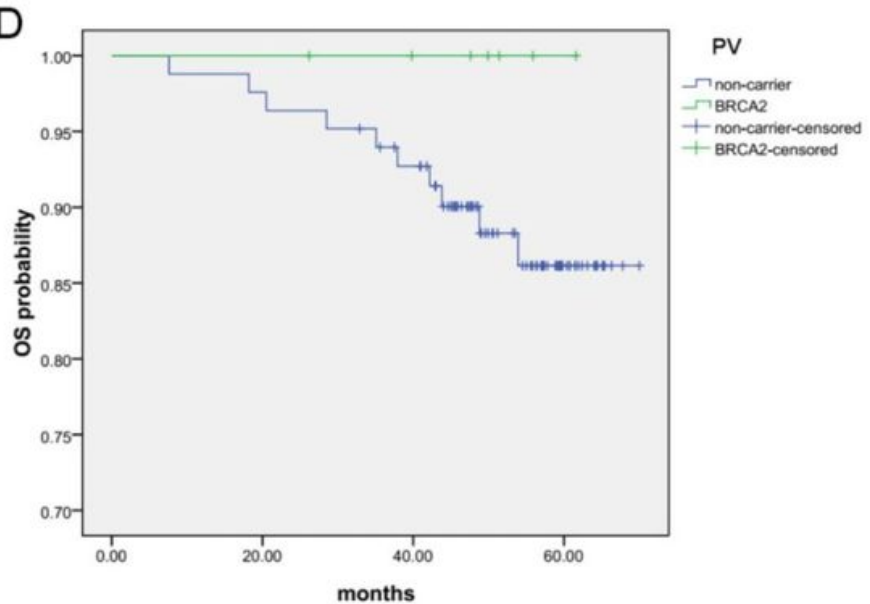

\section{Figure 4}

Kaplan-Meier survival analysis on various BRCA1/2 PVs in TNBC. (A) Progression-free survival (PFS) with and without BRCA1 PV . (B) Overall survival (OS) with and without BRCA1 PV . (C) PFS with and without BRCA2 PV. (D) OS with and without BRCA2 PV.

\section{Supplementary Files}

This is a list of supplementary files associated with this preprint. Click to download.

- FigS1.jpg 